\title{
EchoGéo
}

$17 \mid 2011$

Activités extractives

\section{Quand le géographe laisse sa trace sur le territoire} Introduction

\section{Mathieu Durand}

\section{(2) OpenEdition \\ Journals}

Édition électronique

URL : https://journals.openedition.org/echogeo/12608

DOI : $10.4000 /$ echogeo.12608

ISSN : 1963-1197

Éditeur

Pôle de recherche pour l'organisation et la diffusion de l'information géographique (CNRS UMR 8586)

Référence électronique

Mathieu Durand, "Quand le géographe laisse sa trace sur le territoire », EchoGéo [En ligne], 17 | 2011, mis en ligne le 27 septembre 2011, consulté le 11 août 2021. URL : http://journals.openedition.org/ echogeo/12608; DOI : https://doi.org/10.4000/echogeo.12608

Ce document a été généré automatiquement le 11 août 2021.

EchoGéo est mis à disposition selon les termes de la licence Creative Commons Attribution - Pas d'Utilisation Commerciale - Pas de Modification 4.0 International (CC BY-NC-ND) 


\title{
Quand le géographe laisse sa trace sur le territoire
}

\author{
Introduction
}

Mathieu Durand

1 La géographie est considérée comme une «science qui a pour objet l'espace des sociétés» (Levy et Lussault, 2003), avant de formuler progressivement la notion de territoire. C'est ce territoire qui devient l'enjeu pour les géographes, lorsqu'ils s'intéressent de plus près à l'action publique. Les connaissances qui leur sont spécifiques en terme de fonctionnement du territoire, de logiques spatiales, d'organisation des sociétés, se sont vite avérées intéresser les responsables de la gestion des territoires. Les géographes sont alors devenus les conseillers, les "éminences grises des politiques» (Philipponneau, 1999). Leur intervention dans les affaires publiques a parfois été très visible lorsqu'elle a permis de remodeler les frontières internationales ou d'initier un cycle de plusieurs décennies de réformes décentralisatrices. L'influence des géographes est cependant plus importante au niveau local et régional. C'est en effet sur ces territoires de grande échelle que les liens avec les gestionnaires se font les plus récurrents. Si bien que dans certains cas le géographe a cherché à passer de l'autre côté de la barrière, afin de prendre les rênes de l'action publique et de chercher à obtenir une légitimité politique. Comment évolue l'action des géographes lorsqu'ils ne sont plus simplement en position de conseil mais en position de responsabilité ? Est-ce que ces géographes devenus élus locaux ont une vision spécifique du territoire? En quoi leurs compétences et leurs connaissances leur permettent-elles d'orienter leur action locale? Quels sont les liens entre le métier de géographe et la fonction de responsable politique? Avant d'évoquer l'ensemble de ces points avec un élu local/géographe, revenons sur les accointances entre ces deux rôles.

2 L'un des premiers géographes français contemporains à avoir eu une action directe sur le territoire est Emmanuel de Martonne (Baudel et al., 2001). Successeur de Paul Vidal de la Blache dans l'animation de la récente école de géographie française au début du $\mathrm{XX}^{\mathrm{e}}$ siècle, auteur d'une thèse sur la Valachie, il s'est trouvé être l'un des artisans du dessin des nouvelles frontières en Europe de l'Est. Les responsables politiques de 
l'époque cherchaient à remodeler ce territoire suite à la chute des empires allemands, austro-hongrois et ottomans, après la Première Guerre Mondiale. Les nouveaux États devaient permettre à chaque peuple, selon le principe de la nouvelle Société des Nations, d'être souverain sur son propre territoire. La défaite des trois grands empires permit en effet à toute une série de populations d'obtenir la création de leur ÉtatNation. Ce fut le cas des Slaves du sud, des Polonais, des Slovaques ou des Tchèques. Le second objectif de cette opération était de construire des États puissants, capables de faire face à la résurgence possible de l'Allemagne ou de l'Italie, et même dans une moindre mesure de faire tampon entre l'Europe occidentale et l'empire russe, depuis peu transformé en empire soviétique. Les grandes nations européennes cherchent donc à fixer les frontières des nouveaux États est-européens. Cela sera fait avec les traités de Versailles (1919), de Saint-Germain-en-Laye (1919) et de Sèvre (1920).

C'est dans ce cadre que De Martonne intervient, en tant qu'expert, capable de définir des limites en fonction de critères spatiaux, géopolitiques, culturels et/ou géomorphologiques. Il s'agit là d'une première illustration de l'intervention des géographes à l'échelle large de l'Europe. La géographie, conçue alors comme «science de synthèse " est à même de prendre en compte l'ensemble des dimensions du territoire et des sociétés pour en proposer un redécoupage administratif. La Tchécoslovaquie est alors créée, afin d'unifier les tchèques et les slovaques et de fixer la frontière est de l'Allemagne. La Pologne voit à nouveau le jour. Enfin la Yougoslavie, unifiant les Slaves du sud, auparavant soumis à l'autorité austro-hongroise ou ottomane, est également créée en tant que grand État des Balkans. Il s'avère toutefois, plus de 70 ans plus tard, que malgré certaines caractéristiques communes entre ces populations, ce découpage restait artificiel puisque la plupart de ces pays ont fini par éclater. L'expertise géographique montre ainsi ses limites par rapport à des enjeux politiques ou sociologiques. Elle s'est d'ailleurs depuis cette date sensiblement rapprochée des autres sciences humaines et sociales afin de mutualiser et de compléter les approches.

4 L'un des autres faits marquants des géographes, au-delà de l'aspect géopolitique précédemment cité et des nombreux cas non évoqués dans cette introduction succincte, concerne la création de la notion d'aménagement du territoire. La réorganisation territoriale et les débuts de réflexion autour de l'aménagement du territoire en France ont partiellement été initiés par la publication en 1947 de l'ouvrage du géographe Jean-François Gravier, intitulé « Paris et le désert français ». Suite à la Seconde Guerre Mondiale, la France entre dans une phase de reconstruction et par làmême de réorganisation du territoire national. Jean-François Gravier fait alors le constat d'un déséquilibre criant entre la capitale et le reste du pays. Il s'agit d'un déséquilibre démographique, économique, de poids institutionnel ou de présence des activités de décision. C'est sur cette base que les pouvoirs publics vont mener une réflexion et une action d'aménagement du territoire puis de décentralisation et de rééquilibrage entre les différentes villes françaises.

5 A partir de 1956, avec l'application du " programme d'action régional », la France sera alors divisée en 22 entités administratives, distinctes des régions actuelles. L'objectif est de rapprocher le niveau de décision politique de l'échelon régional, ce qui se fera progressivement sur plusieurs décennies. Ce sont ces territoires, issus entre autres de la réflexion des géographes, qui furent par la suite institués en régions avec une réelle autonomie. Cette décentralisation effective est mise en place par les Lois Deferre de 
1982. Sur la base de ce découpage territorial, on passe alors d'une déconcentration de la décision, toujours aux mains de l'Etat, à une réelle décentralisation. Les Conseils Régionaux sont créés avec des compétences qui s'accrurent par la suite. La décentralisation a progressivement été renforcée, jusqu'à la réforme territoriale de 2010. Celle-ci vise à terme à favoriser une fusion entre les régions et les départements.

Parallèlement à cette décentralisation régionale, fruit de la longue réflexion amenée dans le débat public par l'ouvrage de Gravier de 1947, diverses lois ont promu une décentralisation plus poussée au niveau local. On observe ainsi une diversification des territoires de décision et donc une croissance du nombre d'élus. Ceux-ci ont alors la responsabilité d'agir au niveau des nouveaux groupements de communes, dans les syndicats intercommunaux, communautés de communes ou d'agglomération, Pays, ou enfin avec le récent statut de "métropole». Il s'agit entres autre des lois de 1992, de 1999 ou de 2010. Les géographes ont encore une fois agi de façon importante en appuyant les responsables politiques à travers leurs conseils et leurs analyses sur ces territoires de taille plus modeste. De nombreux élus locaux ou agents territoriaux ont d'ailleurs une formation de géographe, avec une spécialisation de plus en plus poussée vers l'aménagement du territoire, dimension plus appliquée de la discipline.

7 Enfin, la troisième échelle à laquelle interviennent les géographes, est celle de la commune et des territoires urbanisés. L'action directe sur la ville a pendant longtemps été l'exclusivité des architectes. Leur travail reste alors souvent cantonné à des espaces restreints, avec une vision ciblée sur certains espaces ou certaines infrastructures. Les ingénieurs sont ensuite apparus après la révolution hygiéniste de la seconde moitié du $\mathrm{XIX}^{\mathrm{e}}$ siècle pour assainir la ville en travaillant notamment sur les réseaux (eau, assainissement, électricité, voiries, etc.). Seuls les urbanistes ont alors une vision intégrale du territoire urbain, tentant de combiner l'ensemble de ses dimensions. L'entrée des géographes dans la gestion de la ville est alors discrète. Un certain nombre d'urbanistes ont en réalité une formation de géographe s'étant spécialisé sur la question urbaine. Les études de géographie urbaine sont cependant de plus en plus nombreuses à partir de la seconde moitié du XXe siècle (Hertzog et Sierra, 2010), avec notamment les travaux de Georges Chabot et Jacqueline Beaujeu-Garnier (1964), ou encore de Pierre Georges (1952). Ces études permettent de comprendre le fonctionnement des sociétés sur un territoire urbain et les implications spatiales de leurs activités. Le passage vers la dimension appliquée de l'action urbaine est alors très souvent réservé à d'autres disciplines. Les courants portant la " géographie appliquée » ou "géographie active » à partir des années 1960 (Bailly, 2001) revendiquent tout de même cette influence dans la prise de décision au niveau local.

8 Certains géographes ont pourtant réussi à agir de façon directe et concrète sur un territoire, notamment en s'impliquant dans la vie politique. Les élus locaux ayant une formation de géographe ont ainsi une vision particulière de l'espace urbain, percevant l'ensemble de ses dimensions, naturelles ou construites, humaines ou physiques, politiques, sociologiques ou économiques. L'objet de la ville se prête donc tout particulièrement à l'exercice de la géographie. Depuis les premiers géographes conseillant les responsables politiques, tel Hérodote chargé de fonder une cité grecque en Sicile pour Périclès, jusqu'aux géographes se faisant élire sous un régime républicain, la dimension territoriale du champ disciplinaire s'affirme et permet d'avancer dans la gestion territoriale. Les liens étroits existant entre ces deux fonctions, ainsi que la multiplicité des maires de profession "géographe», ont 
d'ailleurs été notés par le Sénat. L'institution est en effet à l'origine d'une conférence, organisée en 1998 et intitulée "Le géographe acteur politique ». Elle a réuni de très nombreux géographes élus locaux. L'un des plus éminents, maire/amateur de géographie, est sans aucun doute actuellement Christian Pierret, maire de la commune de Saint-Dié-des-Vosges et organisateur annuel du Festival International de Géographie. Il déclara lors du colloque de 1998 au Sénat que le géographe et le politique "sont appelés à collaborer, de façon étroite, pour rendre notre monde plus habitable, plus humain, plus solidaire... Bien souvent la géographie a été l'éminence grise du politique, sa bonne et sa mauvaise conscience, celle qui rappelle la nécessité de stratégies régulatrices et l'importance de la légitimation locale. ». Ainsi «le géographe participe, le plus souvent indirectement, à l'action politique, en offrant des choix au décideur» (Philipponneau, 1999). Certains géographes ont alors franchi le cap pour réclamer cette "légitimité locale» auprès des électeurs, afin de faire bénéficier directement la collectivité de leurs compétences et de leurs connaissances. Michel Delebarre, aujourd'hui maire et président de la communauté urbaine de Dunkerque, fut le premier Ministre de la ville en 1990, marquant ainsi la confiance faite à un géographe pour travailler sur les problématiques d'inégalités urbaines. Norbert Métairie, actuel maire et président de la communauté d'agglomération de Lorient, est également géographe de formation.

9 C'est donc dans ce contexte que le texte suivant, retranscrit l'entretien avec Dominique Amiard, géographe et maire de la commune de Cures dans la Sarthe. En rencontrant un responsable politique ayant eu un parcours de géographe, il est possible d'illustrer ces liens entre approche territoriale et action publique.

\section{BIBLIOGRAPHIE}

Bailly A., 2001, Les concepts de la géographie humaine. Paris : Armand Collin, 333p.

Baudelle G., Ozouf-Marignier M.-V., Robic M.-C., 2001. Géographes en pratiques (1870-1945). Le terrain, le livre, la Cité. Rennes : PUR, 390 p.

Chabor G., Beaujeu-Garnier J., 1964. Traité de Géographie urbaine. Paris : Colin.

Georges P., 1952. La ville, le fait urbain à travers le monde. Paris: PUF.

Gravier J.-F., 1947. Paris et le désert français. Paris : Le Portulan, 414 p.

Hertzog A., Sierra A., 2010. Penser la ville et l'urbain, les paradoxes de la géographie française; EchoGéo [En ligne], numéro 12 | 2010, mis en ligne le 31 mai 2010, consulté le 20 septembre 2011. URL : http://echogeo.revues.org/11898

Levy J., Lussault M., 2003. Dictionnaire de la géographie et de l'espace des sociétés. Paris : Belin, 1008 p.

Philipponneau M., 1999. Débat autour du géographe, acteur politique et de la géographie appliquée, avec Jean Bastié, Michel Philipponneau et Christian Pierret. In Le Géographe, acteur politique, Colloquede la Commission de Géographe Appliquée au Sénat, 8 janvier 1998, Acta Geographica, p. 100-105. 


\section{AUTEUR}

\section{MATHIEU DURAND}

Mathieu Durand est maître de conférences à l'Université du Maine, UMR ESO (6590 CNRS).

mathieu.durand@univ-lemans.fr 